\title{
Implementation of Automatic Gas Accident Prevention System using Arduino and GSM
}

\author{
Zamir Chafekar \\ BE (E\&TC) AISSMS \\ IOIT, Pune
}

\author{
Mohd Husain Khan \\ BE (E\&TC) AISSMS \\ IOIT, Pune
}

\author{
Kuldeep Lakra \\ BE (E\&TC) AISSMS \\ IOIT, Pune
}

\author{
S. B. Dhonde, PhD \\ Associate Professor \\ AISSMS IOIT, Pune
}

\begin{abstract}
Gas leakage is a major problem with industrial sector, residential premises and gas powered vehicles like CNG (compressed natural gas) buses, cars etc and this Problem has been the sole reason for many accidents in the past. One of the preventive methods to stop accident associated with the gas leakage is to install gas leakage detection kit at vulnerable places. The aim of this paper is to design and implement automatic gas accident prevention system that can automatically detect and stop gas leakage in vulnerable premises using MQ 5 gas sensor. In this particular gas sensor has been used which has high sensitivity to LPG and natural gases and it detects the leakage of gas if there is any leakage our system will automatically take the preventive measures to avoid fire accidents like turning off the gas supply by turning off the valve and removing the leaked gas from the premises. For extra precautionary method it turns off the main power supply of house. This system also consists of GSM (Global System for mobile communications) module, which alerts by sending SMS to the owner.
\end{abstract}

\section{Keywords}

CNG(compressed natural gas), MQ-5 Gas sensor, Microcontroller (AT328p).

\section{INTRODUCTION}

LPG (Liquefied Petroleum Gas) is a popular cooking fuel. LPG gas is a flammable mixture of hydrocarbon gases (composed of mostly propane and butane) used as a fuel in house appliances and vehicles and in industries. It is odourless gas due to which Ethanethiol is added as powerful odorant, so that leakage can be easily detected. LPG is one of the alternate fuels used now days. Sometimes liquefied petroleum gas is also known as LPG, LP gas, Auto gas etc.

This gas is commonly used for heating appliances, hot water, cooking, and various other purposes also. LPG is also used as an alternate fuel in vehicles due to soaring in the prices of petrol and diesel.

Compressed natural gas (CNG) (methane stored at high pressure) is a fuel which can be used in place of gasoline (petrol), Diesel fuel and propane/LPG. CNG combustion produces fewer undesirable gases than the fuels mentioned above. It is safer than other fuels in the event of a spill, because natural gas is lighter than air and disperses quickly when released. CNG is mainly used for powering vehicles but it is also used for household and industrial purpose as an alternative for LPG gas and other fossil fuels due to is non polluting nature.

Some people have low sense of smell, may or may not respond on low concentration of gas leakage. In such a case, gas leakage security systems become an essential and help to protect from gas leakage accidents. A number of research papers have been published on gas leakage security system.
Embedded system for Hazardous gas detection and Alerting has been proposed where the alarm will be activated immediately, if the gas concentration exceeds normal level.

There have been many accidents that have been caused due to leakage of gas and have caused loss of life and property. Gas leakage detection is not only important but stopping leakage is equally essential. This paper provides a cost effective and highly accurate system, which not only detect gas leakage but also alert (Beep) and turn off the gas supply as well as turn off main power and gas supplies, further it will send an SMS. GSM module is used which alert the user by sending an SMS. In order to provide high accuracy gas sensor MQ-5 has been used.

\section{LITERATURE SURVEY}

Many Systems have been proposed earlier by different authors and researchers and that has helped us to improve the gas safety issue in our every day to day lives.

LPG detection, measurement and booking system. This system presented how to detect the leakage using a gas sensor and book a new cylinder automatically by sending a message to agency. But there was no steps taken to prevent accident in case of leakage. [1]

A System was proposed which detects gas leakage and alerts through alarm and status display besides turning off the gas supply valve. [2]

LPG leakage monitoring and multilevel alerting system was proposed. A system using LPG gas sensor for sensing the leakage and produce the result in audio and visual formats also alerts human via Short Message Service (SMS). But it lacked an automatic prevention system. [3]

A wireless gas leakage \& level detection with auto renewal system was proposed. A system in which gas level is detected and auto booking is done with the gas station also giving the information to the consumer. But it did not deal with the detection and prevention issue. [4]

Real time gas leakage detection using Cloud. This system was proposed in which ZigBee is used to feed real time sensor data over the cloud. The sensor monitors, detects and raises an alarm whenever a gas leak or fire broke out condition is detected. This data is made available at real time feeds over the cloud. But it lacked a self prevention system. [5]

Design and implementation of an economic gas leakage detector was proposed. A system, detecting low and high gas leakage levels and alerts the users by issuing appropriate audio-visual warning signals. The cost involved in developing the system is significantly low and is much less than the cost of gas detectors commercially available in the market. [6]

An android based automatic gas detection and indication robot. A mini mobile robot which is capable of detecting a gas 
leakage in any hazardous places. Whenever there is an occurrence of gas leakage in a particular place the robot immediately read and sends the data to android mobile through wireless communication like Bluetooth. They have developed an android application for android based smart phones which can receive data from robot directly through Bluetooth. The application warns with an indication whenever there is an occurrence of gas leakage and can also control the robot movements via Bluetooth by using text commands as well as voice commands. [7]

A controlling and monitoring system for LPG was proposed. In this system the stove knob is automatically controlled using relay dc motor. Additionally, they have proposed the automatic rebooking of cylinder when the level of gas goes below the normal weight of cylinder. But there was no provision for detection of gas and prevention of fire accident due to gas leakage. [8]

Gas leakage Detection and Smart alerting and prediction using IoT. This system detected the leakage of gas and alerted and also it predicted for any future detection. But there was no provision for prevention and elimination of the leaked gas. [9]

Most of the LPG safety devices present in the market or proposed earlier are mostly based to detect the leakage of gas and home automation like automatic booking etc but there are very few that deal with prevention of accidents that are caused by LPG/CNG gas there are devices that are either designed to prevent leakage or to detect leakage. And here the question arises that what if there is a leakage and how to control it automatically so as to prevent and accidents.

The solution to this problem is our device. Our device not only detects any leakage but automatically stops further leakage and removes the leaked gas to fire accidents and it also alerts the user by sending an SMS.

\section{BLOCK DIAGRAM}

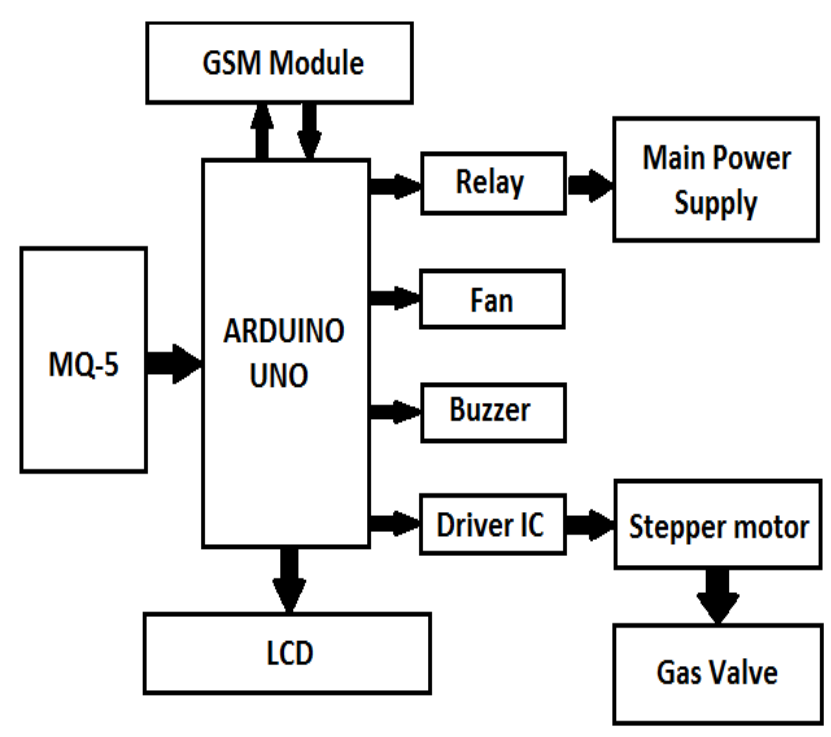

Figure 1: Block Diagram

\subsection{Gas Sensor (MQ5)}

- MQ5 Gas sensor is an electrochemical Gas Sensor more suited to detect and determine LPG concentrations.

- The gas sensor contains an electrode with porous membrane and the gas pass through those membranes and either gets oxidised or reduced and the oxidation produces a current and that current determines the type of gas.

This module has two output possibilities - an analog out (A0) and a digital out (D0). The analog out can be used to detect Gas leakage and to measure volume of Gas leakage in specific units. The digital out can be used to detect Gas leakage and hence trigger a system. The digital out gives only two possible outputs - High and Low. Whenever there is an leakage it gives an digital high out through the digital pin and through the analog pin it gives the concentration of leaked gas.

\subsection{Arduino UNO}

The Arduino UNO is a widely used open-source microcontroller board based on the ATmega328P microcontroller and developed by Arduino.cc. The board is equipped with sets of digital and analog input/output (I/O) pins that may be interfaced to various expansion boards (shields) and other circuits. The board features 14 Digital pins and 6 Analog pins. It is programmable with the Arduino IDE (Integrated Development Environment) via a type B USB cable. It can be powered by a USB cable or by an external 9 volt battery, though it accepts voltages between 7 and 20 volts. It is also similar to the Arduino Nano and Leonardo.

Arduino Uno receives the signal from the gas sensor and then it turn off the main gas supply by the help of stepper motor which is attached to the valve of gas supply controller. The stepper motor is programmed to rotate to the degree till the valve is completely closed. Arduino also stars the buzzer and exhaust fan to remove leaked gas. And it also alerts the owner by sending him or her SMS regarding the leakage.

\subsection{GSM Module}

GSM module is used to send an SMS to the user cell phone. When the gas leakage is detected by the gas sensor, microcontroller sends a signal to GSM module, in which one of the tasks is to send the text SMS. GSM module requires one SIM card. This module is capable to accept any network SIM card. Fig. 3 shows a GSM module IC (Integrated circuit). This module has a unique identity number like mobile phones have. These module works on $12 \mathrm{~V}$ DC supply. We can send SMS and also send a voice message. These SMS or voice messages are saved in the microcontroller memory. Multiple SMSs can also be sends to user, police and fire station etc.

\subsection{Stepper motor}

This system consists of stepper motor driver and stepper motor attached to valve. Stepper motor is connected to the stepper motor driver IC (ULN 2003A). A 12V external DC supply has been given to the stepper motor. The main purpose of the stepper motor is to turn off the main Gas supply. The stepper motor is programmed to rotate to a degree till the valve is completely closed for the valves used in home its 180 degree and for the valves used in industry are 360 degree.

\subsection{Relay}

A relay is an electrically operated switch. Many relays use an electromagnet to mechanically operate a switch, but other operating principles are also used, such as solid-state relays. Relay allows current to pass through it or it is in on state when a high signal is provided to it or otherwise it is in off state. Here the relay is used as a switch for the main power supply of the premises where the device will be used. When there is no leakage high pulse is provided to the relay and when there is an leakage then a low signal is provided that turns off the main power supply. 


\subsection{Exhaust Fan}

It's a Simple DC motor Fan it will be used to remove the Leaked gas.

\section{METHODOLOGY}

As we had earlier seen in the literature survey that there are no automatic device to prevent household disaster caused by LPG and CNG so our device is the solution that problem.

Our device consists of three main parts

- Detection System

- Prevention System

- $\quad$ Alerting System

\subsection{Detection system}

This part consists of a gas sensor and LCD these will continuously monitor the gas concentration.

\subsection{Prevention System}

This part consists of a special type of Gas valve that we will be designing it will be similar to our present regular valves but a stepper motor will attached to its control knob to allow automatic and as well as manual control.

We are also connecting a relay to automatically cut off the main power supply and an exhaust fan will be connected to remove leaked gas.

And all these parts will be interfaced with Arduino Uno which will be controlling the whole device.

\subsection{Alerting system}

It consists of a GSM modem to send an Alert message to the user via SMS.

The detection System gas sensor will continuously monitor for any leakage in gas and if there is any leakage it will send a signal to the microcontroller and then the microcontroller will sound the alarm and turn off the gas supply using an stepper motor that is attached to the valve and remove the leaked gas by turning on the exhaust fan and by using relay it will turn off the main power supply of the house to prevent any fire due to electrical circuit malfunction and then by using GSM module it will also send an SMS to the owner alerting him or her about the incident.

\section{CONCLUSION}

In this paper we have successfully designed and implemented the gas leakage detection system for home safety and industrial applications. This system detects the leakage of the LPG/CNG and alerts the consumer about the leak by sending an SMS and as an emergency measure the system will turn off the valve of Gas supply, while activating the alarm and by using and exhaust fan it removes the leaked gas from the area and also as an extra precautionary measure it turns of the main power supply to prevent fire due to spark or electrical circuit malfunction. Multiple SMS can be sent by changing programming GSM module. This project is implemented using the Arduino UNO based ATmega 328 processor. This system has great scope in the home automation industry this system can be added with extra features that have been discussed in the literature survey like automatic gas booking system and home fire safety system. This system can be modified to be used for industrial purpose especially in the industries where there is emulsion of harmful and flammable gases. The cost involved in developing the system is significantly low and it can be easily made available to the people and the usefulness of this device is immense.

\section{REFERENCES}

[1] Shivalingesh B.M , Ramesh C, Mahesh S.R, Pooja R, Preethi K. Mane, Kumuda S,“ LPG detection, measurement and booking system", volume 1, issue vi, November 2014.

[2] Geeta Loshali, Rohit Basera, Lalit Darmwal And Sachin Varma, "Design \& implementation of lpg gas detector using gsm module", International Journal On Emerging Technologies (special issue ncetst-2017).

[3] Selvapriya , Sathya Prabha, Abdulrahim, Aarthi K C, "LPG leakage monitoring and multilevel alerting system”, International Journal Of Engineering Sciences \& ResearchTechnology.

[4] S.Sivajothi Kavitha1 S. Senthilkumar, "A wireless gas leakage \& level detection with auto renewal system", International Journal Of Advanced Research In Electrical, Electronics And Instrumentation Engineering Vol. 4, issue 4, April 2015.

[5] Sayali Bhogate, Pooja Chavan, Supriya Chavan, Priyanka Doke, Sumita Chandak, "Real time gas leakage detection using Cloud", International Journal Of Innovative Research In Science, Engineering And Technology Vol. 6, issue 4, April2017. website: www.ijirset.com

[6] A.Mahalingam, R. T. Naayagi, N. E. Mastorakis, "Design and implementation of an economic gas leakage detector".

[7] Ch.Manohar Raju And N.Sushma Rani,“An android based automatic gas detection and indication robot", International Journal Of Computer Engineering And Applications, Volume viii, issue i, October 14.

[8] T.Soundarya, J.V .Anchitaalagammai, "Control and monitoring system for Liquefied Petroleum Gas (lpg) detection", International Journal Of Innovative Research In Science, Engineering And Technology Volume 3, special issue 3, March 2014,International conference on innovations in engineering and technology (iciet'14).

[9] Asmita Varma, Prabhakar S, Kayalvizhi Jayavel, Gas Leakage Detection and Smart Alerting and prediction using IoT. Computing and Communications Technologies (ICCCT), 2017 2nd International $\begin{array}{llll}\text { Conference on } & 23-24 & \text { Feb. } & \end{array}$ 